\title{
Aberrant gene expression profiles in hepatocellular carcinoma detected by microdissection
}

\author{
Y.M. Wei ${ }^{1}$,Y.Y. Li ${ }^{2}$, Y.C. Zhang ${ }^{2}$ and Y.Q. Nie $^{2}$ \\ ${ }^{1}$ Department of Blood Transfusion, Guangzhou First People's Hospital, \\ Guangzhou, China \\ ${ }^{2}$ Department of Gastroenterology and Hepatology, \\ Guangzhou First People's Hospital, Guangzhou, China \\ Corresponding authors: Y.Y. Li / Y.M. Wei \\ E-mail: liyyliyy@tom.com / yamingwei@163.com
}

Genet. Mol. Res. 12 (4): 5527-5536 (2013)

Received August 30, 2012

Accepted January 22, 2013

Published November 18, 2013

DOI http://dx.doi.org/10.4238/2013.November.18.3

\begin{abstract}
The aim of this study was to identify genomic aberrations in hepatocellular carcinoma (HCC) by using laser capture microdissection (LCM) combined with microarray analysis. Samples were procured by LCM from HCC and patient-matched normal liver tissue surgically resected from 4 patients. RNA was isolated from the samples and reverse transcribed into cDNA. After 2-cycle linear amplification and 2-color fluorescent labeling, the cRNA was hybridized onto a whole genome microarray. All genes expressed in the normal and HCC samples were counted and analyzed. Differentially expressed genes were identified and the top 10 up and downregulated genes (totally 20 genes) were further evaluated. LCM was able to accurately capture 50-200 cells from HCC and control tissues. The microarray spectrum showed satisfactory detection of HCC-enriched genes. A total of 1361 differentially expressed genes were identified, among which, 607 were upregulated and 754 were downregulated. Among the top 20 up and downregulated genes, 4 genes had not been documented in the literature as being differentially expressed in any tumors. Thus, LCM
\end{abstract}


is an effective approach for identifying aberrantly expressed genes in HCC, and may lead to the discovery of biomarkers for diagnostic and therapeutic applications.

Key words: Laser capture microdissection; Hepatocellular carcinoma; Microarray; Gene expression

\section{INTRODUCTION}

Hepatocellular carcinoma (HCC) is a leading cause of cancer-related death worldwide with the burden of disease expected to increase in coming years (Venook et al., 2010). The development of HCC is a multi-factorial and multi-step process accompanied by expression alterations in critical growth regulatory genes. Identifying the genes involved in this process may lead to new insights into carcinogenesis and enable genetic diagnosis and treatment. The advent of microarray technology with its capacity to simultaneously monitor the expression of thousands of genes in a small input sample provides a unique opportunity for high-throughput genetic analysis. Oligonucleotide microarray has been commonly used to determine the differences in gene expression in tumors and to identify new candidate genes with diagnostic, prognostic, and therapeutic potential (Alizadeh et al., 2001). However, the cellular heterogeneity of input samples has been a significant barrier in the use of microarray technology, as tumor cells may constitute only a minority of the multiple cell types in the tissues analyzed. Analyzing the complete tumor sample without efficiently separating the tumor cells from unrelated cells confounds the true gene expression profile of the tumors. Therefore, special procedures to isolate tumor cells from heterogeneous tissues are mandatory in microecologic studies. Accomplishing this goal is difficult because tissues consist of large numbers of interacting cell populations from the mesenchymal stroma, blood vessels, and necrotic tissues. This problem has now been overcome by laser capture microdissection (LCM), a technique developed in 1996 (Emmert-Buck et al., 1996). LCM enables the procurement of a subpopulation of cells from a specific microscopic region of a tissue section under direct visualization while eliminating cellular contamination from unrelated cells. Intact RNA from the captured cells can be used as starting material to produce cDNA libraries, generate transcript microarray data, perform differential display, and discover tumor-enriched genes (Espina et al., 2009). Therefore, LCM provides a rapid, reliable, and accurate approach for microanalysis methods. Efficiently coupling LCM with microarray analysis has led to the development of sensitive and quantitative methods for the identification of the genetic profiles of tumors from the lung, prostate, and stomach (Wu et al., 2005). However, this method has not been widely used for documenting differences in gene expression in HCC. Only a few such studies have been reported (Yim et al., 2003; Nagai et al., 2004; Wu et al., 2008, 2010). In this study, we identified genes that are differentially expressed in HCC as compared to normal liver tissue using LCM combined with microarray analysis in order to explore the relationship between the genotype and phenotype of HCC in humans.

\section{MATERIAL AND METHODS}

\section{Tissues samples}

Samples and relevant clinical data were obtained from 4 patients with histologically 
confirmed HCC (2 males and 2 females, age 39-56 years) undergoing surgical resection in the Department of Gastroenterological Surgery, Guangzhou First People's Hospital, in July 2009. None of them received chemotherapy or radiation therapy before surgery. Fresh cancer tissue and non-cancerous tissue from the same liver were collected from the surgically resected tissues and immediately frozen at $-85^{\circ} \mathrm{C}$. The study protocols were approved by the ethics committee of Guangzhou First People's Hospital, which is affiliated to Guangzhou Medical University. Written consent was obtained from each patient.

\section{LCM and RNA isolation}

Frozen tissues were sectioned at $6 \mu \mathrm{m}$ with a cryostat section (Thermo Co., USA) at $-20^{\circ} \mathrm{C}$ on polyethylene naphthalate membrane slides. Slides were inverted and mounted onto the microscope stage, where the desired cellular components were excised from the slide by passing the laser through the glass to cut the foil membrane. The sections were allowed to air dry briefly prior to LCM. Gravity facilitated the capture of the dissected cellular components directly into polymerase chain reaction tubes located in a substage tube holder. The dissected cellular components were fixed with RNA stabilization reagent (Qiagen, Germany) for $4 \mathrm{~min}$, and then stained with Mayer's hematoxylin. Pure tumor cell subpopulations and patient-matched non-cancerous populations were microdissected with the use of an LCM system (ASLMD, Leica, Germany) with intensity 70 and speed 8 within 30 min. The paired samples of normal controls from the same sections were also laser captured in the same way. Microdissected cells were stored at $-80^{\circ} \mathrm{C}$ until use. Total RNA extraction was performed using the RNeasy Micro Kit (Qiagen) according to the manufacturer instructions. The extracted RNA was subjected to electrophoresis with the Agilent 2100 Bioanalyzer (Agilent Technologies, USA). The quality of the total RNA was verified using the RNA 6000 Pico Kit with the Bioanalyzer. As recommended by the electrophoresis software of the Bioanalyzer, an index of $\mathrm{A}_{260} / \mathrm{A}_{280} \geq 1.8$ and RNA integrity number $(\mathrm{RIN}) \geq 7$ was set as the quality standard.

\section{RNA amplification and transcription}

Total RNA was used to generate cDNA with a low RNA input linear amplification kit (PLUS, Two-Color, Agilent) and then transcribed again to cRNA. The cRNA was subjected to 2-cycle linear amplification and was fluorescently labeled with $\mathrm{Cy} 3$ for $\mathrm{HCC}$ samples and $\mathrm{Cy} 5$ for normal samples. An ultraviolet spectrophotometer (ND 1000, NanoDrop, USA) was used to determine the concentration and quality of cRNA. Then, $100 \mu \mathrm{L}$ labeled cRNA from each sample was hybridized with a whole genome microarray (Agilent human $4 * 44 \mathrm{~K}$ ) at $65^{\circ} \mathrm{C}$ for $17 \mathrm{~h}$, according to the manufacturer instructions. The microarray was then washed and scanned with a scanner (Agilent GenePix 4000B). The Cy3 and Cy5 fluorescent intensities of all the genes expressed in the 4 paired samples were analyzed and compared to identify differentially expressed genes. The top 10 up and downregulated genes were further researched with Gene Cards (www. genecards.org, Developed at the Crown Human Genome Center, Department of Molecular Genetics, the Weizmann Institute of Science, Israel) and PubMed.

\section{Statistical analysis}

The Agilent Feature Extraction Software (v10.5) was used to normalize the signals of 
$\mathrm{Cy} 3$ and $\mathrm{Cy} 5$. A standard ratio of $\mathrm{Cy} 3 / \mathrm{Cy} 5 \geq 2$ was used as the threshold to identify upregulated genes, and a ratio $\leq 0.5$ was used to identify downregulated genes. Cluster 3.0 Gene Cards and PubMed were used to perform literature reviews for evidence of the expression for the 10 most up and downregulated genes that we identified in HCC. Data were examined with the SPSS 13.0 for Windows statistical package (IBM, USA). $\mathrm{P}<0.05$ was considered to be significant.

\section{RESULTS}

\section{Microdissection and RNA quality control}

HCC and normal control tissues were microdissected with LCM. Pure subpopulations with 50-200 target cells were successfully captured without contamination (Figure 1). The quality of RNA extracted from all samples reached the standard index of $\mathrm{A}_{260} / \mathrm{A}_{280} \geq 1.8$ and RIN $\geq 7$ indicating no contamination from protein or DNA (Table 1). Clear RNA bands upon electrophoresis indicated high purity of RNA without degradation (Figure 2). The $\mathrm{A}_{260} / \mathrm{A}_{280}$ ratio (between 2.0 and 2.1) and the rate of fluorescence incorporation $(>8 \mathrm{pmol} / \mu \mathrm{g}$ ) of cRNA indicated satisfactory purity (Table 2). The high detection rates of the genes are shown in Table 3 , the uniformity of fluorescence intensity is shown in Figure 3, and the coincident trend of gene expression is shown via scatter plots in Figure 4. All products reached quality standards.

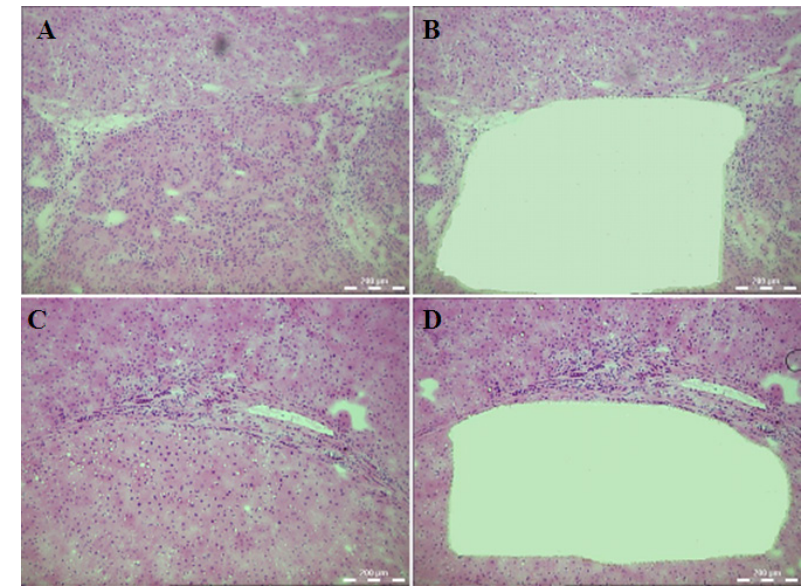

Figure 1. Microdissection of HCC and normal tissuses with LCM (HE x 40). A. HCC (before LCM); B. HCC (after LCM); C. Normal control (before LCM) D. Normal control (after LCM).

Table 1. Quality control of RNA extractions.
\begin{tabular}{lccc}
\hline Code & Concentration $(\mathrm{ng} / \mathrm{uL})$ & $\mathrm{A}_{260} / \mathrm{A}_{280}$ & RIN \\
\hline T1 & 18.00 & 1.92 & 9.50 \\
N1 & 10.00 & 1.97 & 8.20 \\
T2 & 24.00 & 2.01 & 7.80 \\
N2 & 16.00 & 1.93 & 8.00 \\
T3 & 23.00 & 1.98 & 8.30 \\
N3 & 25.00 & 1.90 & 8.20 \\
T4 & 28.00 & 1.95 & 8.40 \\
N4 & 17.00 & 1.94 & 8.80 \\
\hline
\end{tabular}

$\mathrm{A}=$ absorbance $\mathrm{T}=\mathrm{HCC} ; \mathrm{N}=$ normal $; \mathrm{RIN}=\mathrm{RNA}$ integrity number. 


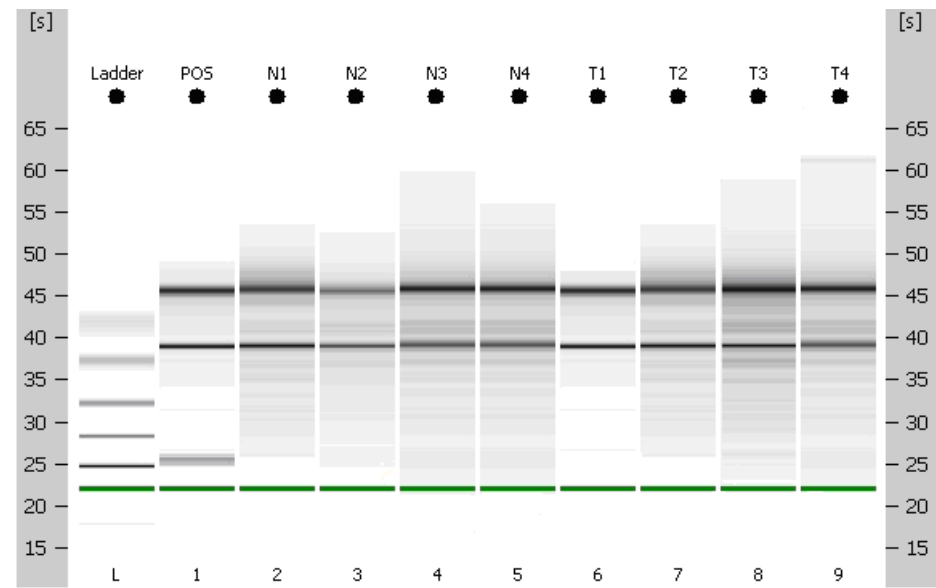

Figure 2. Electropherogram for RNA quality control. $\mathrm{Pos}=$ positive control; $\mathrm{T}=\mathrm{HCC} ; \mathrm{N}=$ normal control.

Table 2. Results of cRNA amplification and fluorescence labeled.

\begin{tabular}{lcccc}
\hline Code & Fluorescence & $\mathrm{A}_{260} / \mathrm{A}_{280}$ & cRNA concentration $(\mathrm{ng} / \mathrm{uL})$ & Fluorescence concentration $(\mathrm{pmol} / \mathrm{uL})$ \\
\hline T1 & Cy3 & 2.03 & 212.70 & 14.30 \\
N1 & Cy5 & 2.07 & 97.60 & 5.00 \\
T2 & Cy3 & 2.08 & 201.00 & 11.70 \\
N2 & Cy5 & 2.10 & 276.70 & 22.80 \\
T3 & Cy3 & 2.05 & 257.40 & 16.70 \\
N3 & Cy5 & 2.03 & 182.70 & 11.10 \\
T4 & Cy3 & 2.05 & 201.70 & 12.80 \\
N4 & Cy5 & 2.03 & 159.10 & 13.20 \\
\hline
\end{tabular}

$\mathrm{A}=$ absorbance; $\mathrm{T}=\mathrm{HCC} ; \mathrm{N}=$ normal.

Table 3. Results of microarray.
\begin{tabular}{lcccc}
\hline Code & Samples (Cy3) & Samples (Cy5) & CV & Detection (\%) \\
\hline 1 & T1 & N1 & 6.45 & 72.12 \\
2 & T2 & N2 & 8.27 & 74.91 \\
3 & T3 & N4 & 4.68 & 69.65 \\
4 & T4 & 5.99 & 70.30 \\
\hline
\end{tabular}

$\mathrm{CV}=$ coefficient of variation.

\section{Differentially expressed genes}

The expression of a total of 1361 genes was detected to be significantly altered $(\mathrm{P}<$ $0.05)$ in the 4 pairs of HCC and control samples. Among these, 607 were upregulated and 754 were downregulated. The differentially expressed genes were ranked and classified according to their signal log ratio. The 20 genes showing the greatest changes in expression, i.e. the top 10 upregulated and downregulated genes were researched through literature searches via the Gene Cards and PubMed databases. Among them, 5 genes were previously shown to be related to $\mathrm{HCC}$ and 7 were validated as being associated with other tumors. The remaining 4 genes had not been reported as differentially expressed genes in any tumors (Tables 4 and 5). 


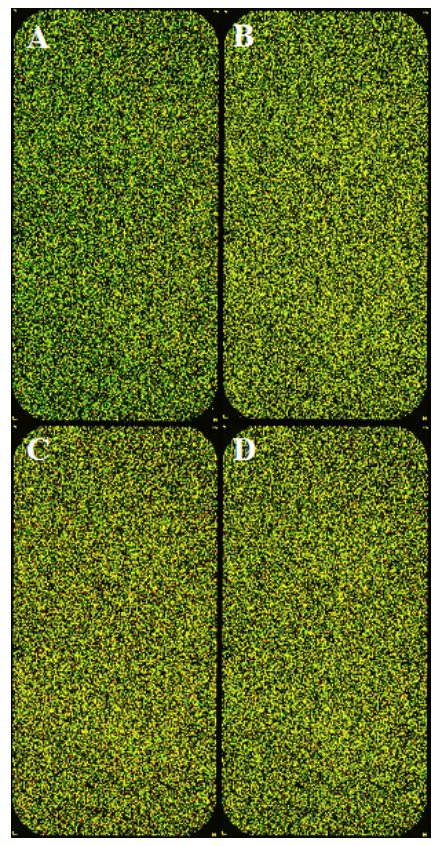

Figure 3. Two-color fluorescent (Cy3, Cy5) labeled cRNA hybridization. A. HCC 1 and normal 1; B. HCC 2 and normal 2; C. HCC 3 and normal 3; D. HCC 4 and normal 4.
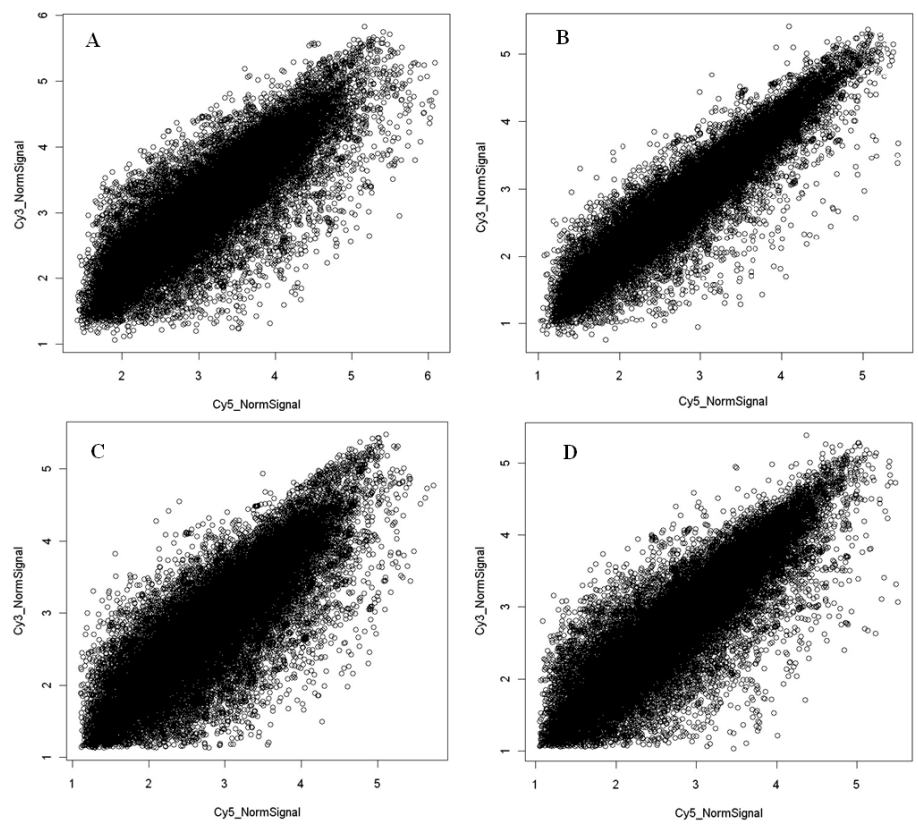

Figure 4. Scatter diagrams of differential gene profiles of HCC and normal control A. HCC 1 and normal 1; B. HCC 2 and normal 2; C. HCC 3 and normal 3; D. HCC 4 and normal 4. 
Table 4. Top ten upregulated genes in HCC.

\begin{tabular}{llcl}
\hline Gene symbol & Unigene code & Ratio & Name and description \\
\hline TACSTD1 $^{\text {a }}$ & Hs.692 & 23.2529 & Tumor-associated calcium signal transducer 1 \\
TTK $^{\text {a }}$ & Hs.169840 & 27.3729 & TTK protein kinase \\
NEK2 $^{\text {b }}$ & Hs.153704 & 32.6949 & NIMA serine/threonine related kinase 2 \\
CTNND2 $^{\text {b }}$ & Hs.314543 & 28.4809 & Catenin (cadherin-associated protein), delta 2 (neural plakophilin-related arm-repeat protein) \\
CENPA $^{\text {b }}$ & Hs.1594 & 23.7030 & Centromere protein A, 17 kDa \\
CCR6 $^{\text {b }}$ & Hs.46468 & 21.3423 & Chemokine (C-C motif) receptor 6 \\
CDCA7 $^{\text {b }}$ & Hs.470654 & 19.1093 & Cell division cycle associated 7 \\
CDCA1 $^{\text {b }}$ & Hs.234545 & 17.2919 & Cell division cycle associated 1 \\
DEPDC1B $^{\text {c }}$ & Hs.482233 & 24.6761 & DEP domain containing 1B \\
ASPM $^{\text {c }}$ & Hs.121028 & 22.0722 & Asp (abnormal spindle)-like, microcephaly associated (Drosophila) \\
\hline
\end{tabular}

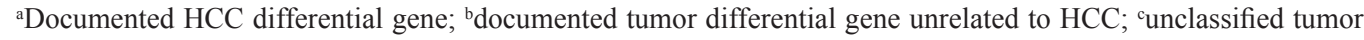
differential gene.

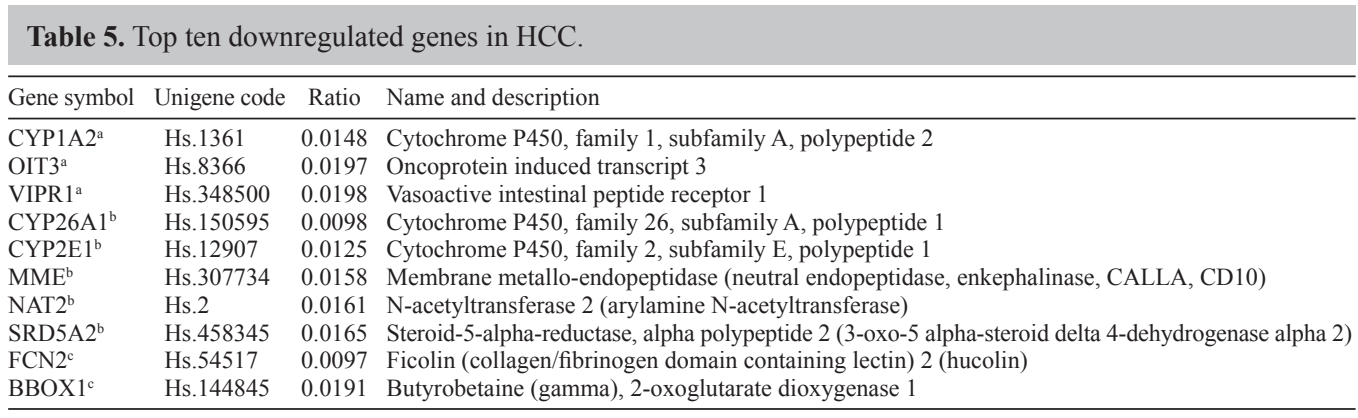

${ }^{a}$ Documented HCC differential gene; ${ }^{b}$ documented tumor differential gene unrelated to HCC; ${ }^{c}$ unclassified tumor differential gene.

\section{DISCUSSION}

LMD is a powerful technology for isolating specific cells or cell groups for further analysis. It eliminates the problems of cell-type heterogeneity within tissue samples by providing homogeneous cell populations for analysis. The technique allows highly specific comparisons of cancer cells with normal cells from the same tissue sample. Microarray analysis offers a new avenue to understand the biological diversity of cells (Alizadeh et al., 2001). Most previous gene expression studies have made use of whole tumor tissues. Although this data is comprehensive, it is limited by the fact that the tumor samples were contaminated with unrelated cells (Okabe et al., 2001). Therefore, special procedures to isolate cancer cells from heterogeneous tissues are necessary. LCM enables the isolation of pure cell populations from a native tissue environment. The percentage of cell contamination with LCM is estimated to be less than $0.3 \%$ (Nakamura et al., 2004). In the past, the amount of RNA procured from cancer cells isolated by LCM was not sufficient for hybridization with a cDNA microarray. Recent advances in linear RNA amplification have provided the key to resolve this problem. This technique not only provides sufficient RNA for further analyses but also preserves overall genetic information (Aoyagi et al., 2003).

The combination of LCM with microarray analysis has been successfully used to identify the molecular subtypes of many different tumors (Wu et al., 2005). Several animal and human studies of HCC have been published that report the differential expression of genes in 
HCC as compared to same tissues with other liver disease. Nagai et al. identified 72 upregulated and 57 downregulated genes in liver-cirrhosis nodules as compared to HCC tissue from a single patient. Among them, the expression of 31 upregulated and 7 downregulated genes were positive both in HCC and liver-cirrhosis nodules (Nagai et al., 2004). Wu et al. showed that the NS5A protein downregulated the expression of the mitotic spindle protein ASPM and induced an aberrant mitotic cell cycle, which was associated with chromosome instability and subsequently HCC (Wu et al., 2008). Wu et al. studied human osteopontin positive and negative HCC tissues and found some tumor-related genes, such as SMR 3B, MUC7, EPHA5, $S P P 1$, and $C L D N 10$ to have significantly higher expression in osteopontin positive HCC than in osteopontin negative HCC (Wu et al., 2010). Yim et al. identified 855 differentially expressed genes in HCC and hepatic adenomas using a transgenic rat model. In total 855 genes, 71 were positive expressed in both HCC and hepatic adenomas, and among 71 positive genes, 28 of them were upregulated in both tissues and 41 were downregulated in both tissues (Yim et al., 2003).

In this study, we generated new data that adds to our understanding of $\mathrm{HCC}$ and complements the data from the studies described above. We successfully identified $1361 \mathrm{HCC}-$ enriched genes with more than $95 \%$ accuracy. In order to further understand our results, we performed literature searches for the top 10 up and downregulated genes using the Gene Cards and PubMed databases. Of these 20 genes, 16 had a known association with HCC (Reubi et al., 2000; Okabe et al., 2001; Kim et al., 2004; Chen et al., 2006) or other tumors (Hein, 2002; Tomonaga et al., 2003; Agundez, 2004; Lu et al., 2005; Osthus et al., 2005; Hayward and Fry, 2006; Bethke et al., 2007). Therefore, our results are consistent with the literature. Of the 16 genes associated with HCC or other tumors, the following genes were the most interesting: 1) TACSTD1 (tumor-associated calcium signal transducer 1): this gene encodes the epithelial cell adhesion molecule EpCAM, which is expressed in the hepatic stem cells of embryos. TACSTD1 shows the most significantly altered level of gene expression in adult cirrhosis and HCC (Lu et al., 2005), 2) NAT2 (N-acetyltransferase 2): this gene encodes an important enzyme involved in the biotransformation of carcinogens and exhibits genetic polymorphisms. NAT2 acetylator genotypes are important modifiers of human cancer susceptibility (Hein, 2002), 3) NEK2 (NIMA-related serine/threonine kinase): this gene contributes to chromosome instability and is abnormally expressed in a wide variety of human cancers (Reubi et al., 2000). P450 family, including CYP1A2 $2^{\mathrm{a}}, \mathrm{CYP} 26 \mathrm{~A} 1^{\mathrm{b}}$ and CYP2E1 ${ }^{\mathrm{b}}$ downregulated in these HCC tissues, have been reported to be related to the susceptibility of many types of cancers (Agundez, 2004; Bethke et al., 2007).

The remaining 4 differentially expressed genes, which were not previously reported in the literature to be associated with carcinogenesis, were of particular interest. The discovery of these 4 genes is novel and that these genes may correspond to the different histological phenotypes of HCC. If so, they may be useful as candidate genes for the diagnosis and treatment of HCC. The ASPM (abnormal spindle-like microcephaly) gene that encodes a mitotic spindle protein is widely expressed in fetal and proliferating adult tissues (Kouprina et al., 2005; Wu et al., 2008). The SCFCN2 (Ficolin 2) gene encodes ficolin-2 (L-ficolin), which regulates innate immunity (Munthe-Fog et al., 2007). BBOX1 (butyrobetaine 2-oxoglutarate dioxygenase 1) encodes an enzyme responsible for the biosynthesis of 1-carnitine, a key molecule in fatty acid metabolism. Its activity has been detected in the kidney, liver, and brain (Rigault et al., 2006). The $D E P D C 1 B$ (DEP domain containing 1B) gene encodes the receptor-like protein- 
tyrosine-phosphatase (PTP), which may contribute to the inhibition of cell growth (Ostman et al., 1994). These findings suggest that genes associated with tissue proliferation, metabolism, and immune response might play roles in the development of HCC.

In this study, we extracted RNA from microdissected human HCC and patient-matched normal liver cells for the examination of their gene expression profiles. We demonstrated that this strategy is a rapid and efficient way to identify the transcriptional signature of HCC. Further studies are needed to determine the potential value of the genes identified here as novel biomarkers for diagnostic and therapeutic applications.

\section{ACKNOWLEDGMENTS}

Research supported by the National Natural Science foundations of China (\#81070385), Guangzhou Medicine Key Projects (\#201102A212011), and Foundation from Guangzhou Science and Technology Bureau, China (\#2006Z3-10031).

\section{REFERENCES}

Agundez JA (2004). Cytochrome P450 gene polymorphism and cancer. Curr. Drug Metab. 5: 211-224.

Alizadeh AA, Ross DT, Perou CM and van de Rijn M (2001). Towards a novel classification of human malignancies based on gene expression patterns. J. Pathol. 195: 41-52.

Aoyagi K, Tatsuta T, Nishigaki M, Akimoto S, et al. (2003). A faithful method for PCR-mediated global mRNA amplification and its integration into microarray analysis on laser-captured cells. Biochem. Biophys. Res. Commun. 300: 915-920.

Bethke L, Webb E, Sellick G, Rudd M, et al. (2007). Polymorphisms in the cytochrome P450 genes CYP1A2, CYP1B1, CYP3A4, CYP3A5, CYP11A1, CYP17A1, CYP19A1 and colorectal cancer risk. BMC Cancer 7: 123.

Chen X, Wang H, Xie W, Liang R, et al. (2006). Association of CYP1A2 genetic polymorphisms with hepatocellular carcinoma susceptibility: a case-control study in a high-risk region of China. Pharmacogenet. Genomics 16: 219-227.

Emmert-Buck MR, Bonner RF, Smith PD, Chuaqui RF, et al. (1996). Laser capture microdissection. Science 274: 9981001.

Espina V, Wulfkuhle J and Liotta LA (2009). Application of laser microdissection and reverse-phase protein microarrays to the molecular profiling of cancer signal pathway networks in the tissue microenvironment. Clin. Lab. Med. 29: $1-13$.

Hayward DG and Fry AM (2006). Nek2 kinase in chromosome instability and cancer. Cancer Lett. 237: 155-166.

Hein DW (2002). Molecular genetics and function of NAT1 and NAT2: role in aromatic amine metabolism and carcinogenesis. Mutat. Res. 506-507: 65-77.

Kim JW, Ye Q, Forgues M, Chen Y, et al. (2004). Cancer-associated molecular signature in the tissue samples of patients with cirrhosis. Hepatology 39: 518-527.

Kouprina N, Pavlicek A, Collins NK, Nakano M, et al. (2005). The microcephaly ASPM gene is expressed in proliferating tissues and encodes for a mitotic spindle protein. Hum. Mol. Genet. 14: 2155-2165.

Lu Q, Dobbs LJ, Gregory CW, Lanford GW, et al. (2005). Increased expression of delta-catenin/neural plakophilin-related armadillo protein is associated with the down-regulation and redistribution of E-cadherin and p120ctn in human prostate cancer. Hum. Pathol. 36: 1037-1048.

Munthe-Fog L, Hummelshoj T, Hansen BE, Koch C, et al. (2007). The impact of FCN2 polymorphisms and haplotypes on the Ficolin-2 serum levels. Scand. J. Immunol. 65: 383-392.

Nagai H, Terada Y, Tajiri T, Yabe A, et al. (2004). Characterization of liver-cirrhosis nodules by analysis of geneexpression profiles and patterns of allelic loss. J. Hum. Genet. 49: 246-255.

Nakamura T, Furukawa Y, Nakagawa H, Tsunoda T, et al. (2004). Genome-wide cDNA microarray analysis of gene expression profiles in pancreatic cancers using populations of tumor cells and normal ductal epithelial cells selected for purity by laser microdissection. Oncogene 23: 2385-2400.

Okabe H, Satoh S, Kato T, Kitahara O, et al. (2001). Genome-wide analysis of gene expression in human hepatocellular carcinomas using cDNA microarray: identification of genes involved in viral carcinogenesis and tumor progression. Cancer Res. 61: 2129-2137. 
Osthus RC, Karim B, Prescott JE, Smith BD, et al. (2005). The Myc target gene JPO1/CDCA7 is frequently overexpressed in human tumors and has limited transforming activity in vivo. Cancer Res. 65: 5620-5627.

Ostman A, Yang Q and Tonks NK (1994). Expression of DEP-1, a receptor-like protein-tyrosine-phosphatase, is enhanced with increasing cell density. Proc. Natl. Acad. Sci. U. S. A. 91: 9680-9684.

Reubi JC, Laderach U, Waser B, Gebbers JO, et al. (2000). Vasoactive intestinal peptide/pituitary adenylate cyclaseactivating peptide receptor subtypes in human tumors and their tissues of origin. Cancer Res. 60: 3105-3112.

Rigault C, Le Borgne F and Demarquoy J (2006). Genomic structure, alternative maturation and tissue expression of the human BBOX1 gene. Biochim. Biophys. Acta 1761: 1469-1481.

Tomonaga T, Matsushita K, Yamaguchi S, Oohashi T, et al. (2003). Overexpression and mistargeting of centromere protein-A in human primary colorectal cancer. Cancer Res. 63: 3511-3516.

Venook AP, Papandreou C, Furuse J and de Guevara LL (2010). The incidence and epidemiology of hepatocellular carcinoma: a global and regional perspective. Oncologist 15 (Suppl 4): 5-13.

Wu JC, Sun BS, Ren N, Ye QH, et al. (2010). Genomic aberrations in hepatocellular carcinoma related to osteopontin expression detected by array-CGH. J. Cancer Res. Clin. Oncol. 136: 595-601.

Wu MS, Lin YS, Chang YT, Shun CT, et al. (2005). Gene expression profiling of gastric cancer by microarray combined with laser capture microdissection. World J. Gastroenterol. 11: 7405-7412.

Wu SC, Chang SC, Wu HY, Liao PJ, et al. (2008). Hepatitis C virus NS5A protein down-regulates the expression of spindle gene Aspm through PKR-p38 signaling pathway. J. Biol. Chem. 283: 29396-29404.

Yim SH, Ward JM, Dragan Y, Yamada A, et al. (2003). Microarray analysis using amplified mRNA from laser capture microdissection of microscopic hepatocellular precancerous lesions and frozen hepatocellular carcinomas reveals unique and consistent gene expression profiles. Toxicol. Pathol. 31: 295-303. 